\title{
Energy Efficient and Latency Aware TDM-PON for Local Customer Internetworking
}

\author{
S.H. Shah Newaz ${ }^{1}$, Alaelddin Fuad Yousif Mohammed ${ }^{1}$, Gyu Myoung Lee ${ }^{1,2}$, Jun Kyun Choi ${ }^{1}$ \\ ${ }^{1}$ Korea Advanced Institute of Science and Technology (KAIST) \\ Daejeon, 305-732, South Korea. \\ ${ }^{2}$ School of Computing and Mathematical Sciences, Liverpool John Moores University \\ Liverpool, United Kingdom. \\ Email: newaz@kaist.ac.kr, alaelddin@kaist.ac.kr, G.M.Lee@ljmu.ac.uk, jkchoi59@kaist.edu.
}

\begin{abstract}
In this paper, we propose a novel energy efficient solution for TDM Passive Optical Networks (TDM-PONs), which is a widely used access network technology, to facilitate local customer internetworking. Here, our prime objectives are to ensure traffic latency among different customers connected through TDM-PON and maximize energy saving performance of Optical Network Units (ONUs), which are TDM-PON equipment situated at customer premises. To do so, we devise a novel sleep management protocols for ONUs. In our proposal, when some ONUs need to exchange traffic, they maintain sleep mode as a group in order to reduce traffic latency among the customers attached with those ONUs. To date, considering traffic forwarding between access and core network entities, researchers have come up with several solutions to manage sleep mode in ONUs in order to improve PON energy saving. However, to the best of our knowledge, how ONUs can manage sleep mode and at the same time satisfy latency requirements while managing data flows among them has been paid little attention. The effectiveness of our solution is validated and compared with other alternative approach by simulation. Simulation results demonstrate that proposed solution reduces inter-ONU traffic delay noticeably compared to other solution while providing satisfactory energy saving performance of ONUs.
\end{abstract}

Index Terms-TDM-PON internetworking, energy saving, Fog computing, traffic latency, smart grid, IoT.

\section{INTRODUCTION}

With the increasing concern on the influence of energy consumption of ICT due to rapid network expansion and stupendous growth of network traffic, many research initiatives have been carried out to make network energy efficient while not affecting Quality of Service (QoS) of networks (in 2002, communication network contributed in producing 64 megatons of $\mathrm{CO}_{2}$ [2]). Research findings impart that access networks are significantly large contributors for total amount of energy consumed by ICT; however, they are underutilized most of the time. The most common approach for saving energy of access network equipment is sleep mode (a node turns off its power hungry components during a defined amount of time).

Among different access technologies (e.g. Passive Optical Networks (PON), WiMAX, WiFi), PON appears as a very promising technology as it provides huge data rate up to the order of Gbps and consumes much less power than other access network technologies like WiMaX [3]. However, there is still room to improve its energy saving performance [3].
Energy efficiency and latency have very strong trade off relationship which should be taken into consideration in any energy efficiency aware traffic forwarding proposal. In particular, there are some applications that require stringent delay requirements [1], [3] (e.g. voice traffic, gaming, and smart mobile applications (smart connected vehicles)).

The necessity of data forwarding among edge and access nodes (e.g. end users, local computation servers) is increasingly becoming the subject of attention these days. Indeed, distance between end points can play a crucial role in determining how much delay a particular flow might experience. In order to ensure delay requirements of delay sensitive applications, researchers are sought to use local computational and storage resources. For example, in 2012, Cisco introduced the concept of Fog computing [4], where servers are put close to the end users to serve local computational support. In Fog computing, different distributed Fog nodes and end users are connected through different access network technologies (e.g. PON, WiMAX, WiFi). In addition, in a local area network domain, ends users might need to exchange traffic (e.g. teleconferencing, information broadcasting) among themselves [7].

Based on the aforementioned discussion, we argue that the necessity of data forwarding in a local area domain is becoming a subject of growing attention. On the other hand, energy efficiency improvement of access network equipment (e.g. ONUs, Base Stations) has been an important research focus of industries and academia over the last several years. Therefore, it is timely to put research effort on developing a solution that can facilitate local customer (e.g. end users, Fog servers) internetworking efficiently and minimize energy consumption of access network equipment at the same time. In this paper, we propose an energy efficient and latency aware local customer internetworking solution based on TDM-PON. Note that, there are some existing works (e.g. [5]-[7]) that study only how local customer traffic can be forwarded efficiently in a TDM-PON. However, energy saving performance of TDM-PON equipment (e.g. Optical Network Units (ONUs)) is not a major concern in those works.

Here, in this paper, we propose a novel sleep management protocol for ONUs. In our proposal, when a group of ONUs has traffic to forward among local customers, ONUs belong 


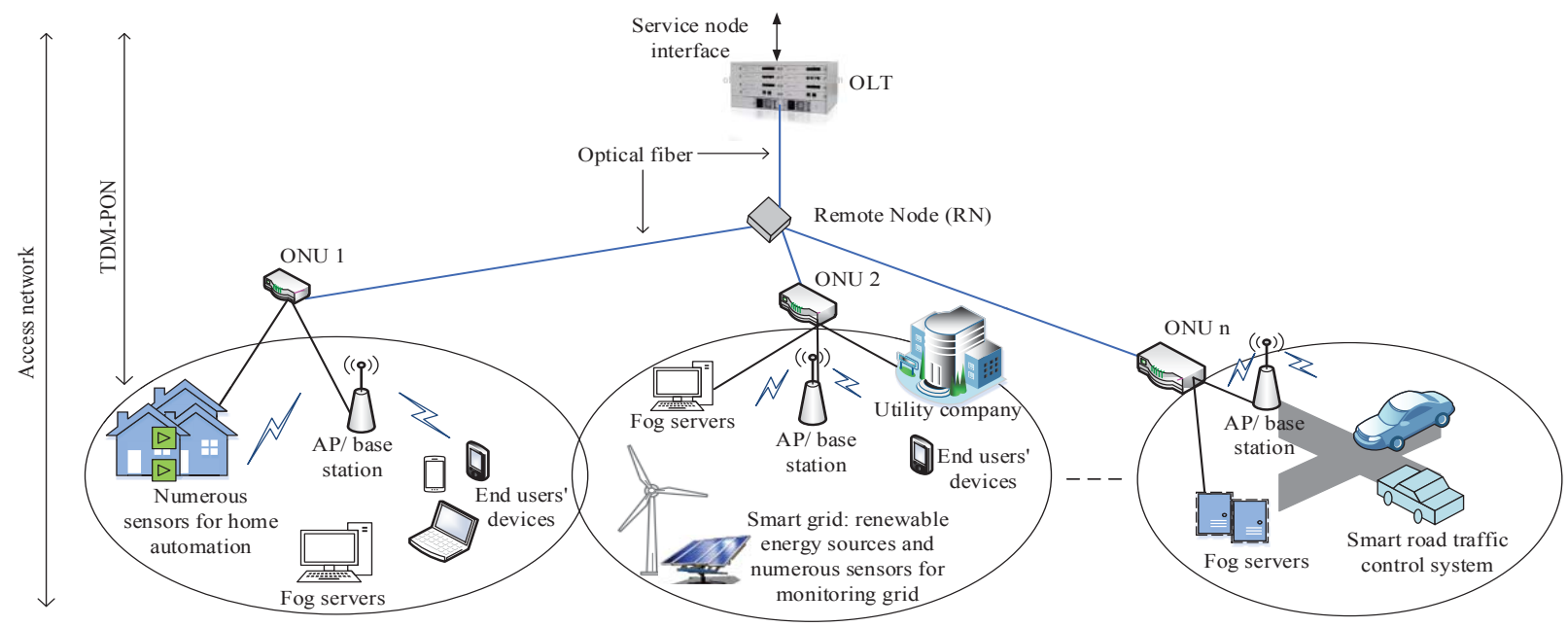

Fig. 1. TDM-PON based local customer internetworking architecture: serving numerous applications of customer premises.

to that group manage sleep mode synchronously in order to ensure that the delay requirement for the customers attached with those ONUs is not violated. The rest of the paper is organized as follows. Section II presents related work. Section III introduces overall system model for our proposed solution. Section IV describes the proposed traffic latency aware and energy efficient local customer internetworking solution. Section $\mathrm{V}$ presents performance evaluation results of our solution. Finally, section VI concludes this paper.

\section{RELATED WORK}

In this section, we first briefly describe the operation of a TDM-PON (e.g. Ethernet PON (EPON) [9]), and then we explain how it supports local customer internetworking. In addition, this section identifies the importance of local customer internetworking in a PON.

\section{A. TDM-PON Operation and Local Traffic Internetworking:}

A TDM-PON has a point to multipoint optical fiber based networking architecture (a typical architecture forms a tree-like topology) [3], [8]. The main components of a TDM-PON are: (1) Optical Line Terminal (OLT) which is situated at a central office, (2) $l * N$ passive star coupler (SC), which does not require any power supply, and (3) ONUs, which are installed at the customer premises, connecting several end users. Typical distance between an OLT and ONU is less than $20 \mathrm{~km}$. In a TDM-PON, the OLT, which is the centralized intelligence of a TDM-PON, forwards downlink traffic in a broadcast manner. Whereas, in case of uplink transmission (an ONU to the OLT), each of the ONUs connected with the OLT through optical fiber is assigned a dedicated uplink transmission slot. A TDMPON uses two separate wavelengths for uplink and downlink transmission [3], [8], [9].

End users served by different ONUs, which are connected with a single TDM-PON, might need to share resources, including contents, computational support and storage support.
In such cases, a TDM-PON should be able to emulate or operate as a local area network [8]. One possible approach considered earlier is that when ONUs under a single TDM-PON need to forward traffic among them, a sender ONU should first forward to the OLT, and then the OLT forwards these traffic to the destination ONU [8]. In this paper, we named this approach as Typical TDM-PON Based Internetworking (TTBI) approach. It is because the TTBI does not require any additional component to be deployed. However, in case of $1 \mathrm{G}$ TDM-PON, using TTBI approach conventional access service might experience bandwidth scarcity as LAN emulation (inter ONU traffic forwarding) uses a portion of bandwidth from downlink and uplink optical channels. Taking this limitation into account, several other local customer internetworking solutions have been introduced (e.g. [5]-[7], [10]).

Authors in [10] proposed a physical-layer based solution for local customer internetworking in TDM-PONs. In this solution, using optical switches, a TDM-PON is virtually divided into two independent sub-networks (a separate broadband access for conventional access service and the other for local customers). Another hardware based solution is proposed in [7]. In this solution, three separate wavelengths are dispensed for downlink $\left(\lambda_{D L}\right)$, uplink $\left(\lambda_{U L}\right)$, and local customer internetworking $\left(\lambda_{L C}\right)$, associated traffic. Additionally, in [7], authors came up with a new ONU architecture, where unlike a conventional ONU [8], an ONU requires a tunable transmitter and receiver. In their solution, when an ONU needs to send traffic to the OLT of a TDM-PON, the ONU's tunable transmitter generates $\lambda_{U L}$ optical signal. Whereas, this transmitter produces optical signal with $\lambda_{L C}$ when the ONU has traffic to send to any other ONUs under the same TDMPON. Note that a tunable transmitter cannot generate $\lambda_{U L}$ and $\lambda_{L C}$ at the same time. Therefore, an ONU needs to switch its transmission mode based on requirement. Similarly, an ONU's tunable receiver needs to switch from one mode to another 
mode time to time (receive $\lambda_{D L}$ from the OLT and $\lambda_{L C}$ from other ONUs).

The major shortcomings of the hardware based solutions are that they require additional hardware and software implementation in a PON. Note that a large number of ONUs are already deployed around the world. Therefore, network operators who have already deployed PONs, adopting hardware based solutions (e.g. [5]-[7]) is neither a cost-effective nor a practical approach. As mentioned earlier that TTBI approach could cause bandwidth scarcity for conventional access service. However, this might be a case for a TDM-PON with low data rate capability (e.g. $1 \mathrm{G}$ EPON). The fact is that over the last several years PON data rate has increased by several folds. For example, 10G EPON (IEEE 802.3av [11]) has been standardized in 2009 and already installed widely. Additionally, researchers are putting effort to further improve PON data rate (Huawei tested 40G PON prototype in 2011 [12]). Therefore, hardware based local customer internetworking solutions are very less likely to be adopted in high speed TDM-PONs (e.g. 10G EPON). Whereas, in TTBI approach, which requires only software update in a conventional TDMPON to support local customer internetworking, would not impose much deployment overhead. Therefore, undeniably, TTBI approach would be a promising and an attractive solution to the PON operators in the coming days.

\section{B. The Rising Importance of Local Customer Internetworking:}

There are several applications where latency is a prime concern (e.g. voice conversation, video conferencing, online gaming). Furthermore, over the last several year, the growth of Internet of Things (IoT) uses has increased significantly (new uses of IoT are emerging as time goes by). For example, there are numerous uses of IoT in home automation, industrial automation, distributed control system (smart grid, connected rail, smart traffic light systems [1]).

In a smart traffic light system, different sensors need to continuously monitor several important factors, including distance and speed of vehicle, and presence of pedestrians around road crossings [1]. To ensure safe road traffic control system, local control loop subsystem should make decision and execute different operations within a few milliseconds [1]. In [13], authors came up with wearable brain-computer interface (BCI) system which is developed to make realtime prediction of human cognitive state. In their BCI system, latency is one of the key factors. Therefore, authors dispensed distributed computing approach (multi-tier Fog and Cloud Computing) along with sensors and state-of-art wireless communication technology. Authors claim that Fog servers can play significantly important role in actualizing real-time human-computer interactions. Some applications in a smart grid require stringent delay requirement [14]-[16]. To run smart grid efficiently, reliable communication infrastructure that can support strict delay requirements for some missioncritical applications is increasingly important. In a smart grid, different utility applications have different level delay requirements [14]. For example, delay requirement for teleprotection and synchrophasor applications are about $10 \mathrm{~ms}$ and $20 \mathrm{~ms}$, respectively [14].

In these aforementioned applications, it is important to reduce the distance between end user devices (e.g. mobile, sensors) and the decision center (e.g. local control loop subsystem, smart grid utility companies); so that, latency requirements of different utility applications can be satisfied [15]. One of the promising solutions is distributed computing paradigm in which end users' nearby available online computing resources are assigned computation tasks (known as the Fog servers) [13]. Therefore, it would be a very likely scenario where a local customer (end users' devices, IoTs) connected with an ONU might require computational support from a Fog server which is connected with an another ONU within the same TDM-PON. Similarly, several computational servers connected with different ONUs might need to exchange traffic. Therefore, it can be claimed that in the near future local customer internetworking in a TDM-PON will be significantly important.

\section{Proposed EnERGy-EFFICIENT LATENCY AwARE LOCAL CUSTOMER INTERNETWORKING SCHEME}

This section presents our proposed energy-efficient and latency aware local customer internetworking scheme. Our proposal relies on TTBI (Typical TDM-PON Based Internetworking) approach, which has been introduced in Section II. The purpose of using TTBI is that this approach requires only software level modification in existing TDM-PON architecture (it would not impose much deployment overhead for operators). From now on, we will refer to this proposed solution as Energy-efficient Latency-aware TTBI (EL-TTBI) scheme.

\section{A. EL-TTBI System Model:}

In our proposal, a TDM-PON manages sleep mode to improve its energy saving performance. Note that TTBI approach reported in [8] does not use sleep mechanism. In our solution, we assume that an ONU can have two modes: Active mode and Sleep mode. We use the ONU architecture considered in [17]. We assume that in the customer premises, there are different types of traffic sources including smart grid utility applications, smart traffic system, and on-line gaming. In addition, there are Fog servers available in access segments (customer premises) for computational supports for the resource constraints end devices as depicted in Fig. 1.

Figure 2 presents a typical TDM-PON architecture which is considered for our proposed EL-TTBI scheme. This figure shows that when ONUs have traffic to share, the traffic should travel through the OLT (here, ONUs do not have any direct optical connection). In the downlink direction, the OLT uses optical signal with $\lambda_{D L}$ for broadcasting downlink traffic, whereas in the uplink direction ONUs use $\lambda_{U L}$ for uplink transmission during their assigned transmission slots. Here, we consider that the OLT is in charge of maintaining sleep mode in ONUs, as considered in [3], [18]. That is, the OLT knows when a particular ONU needs to stay active to receive and/or transmit traffic. 


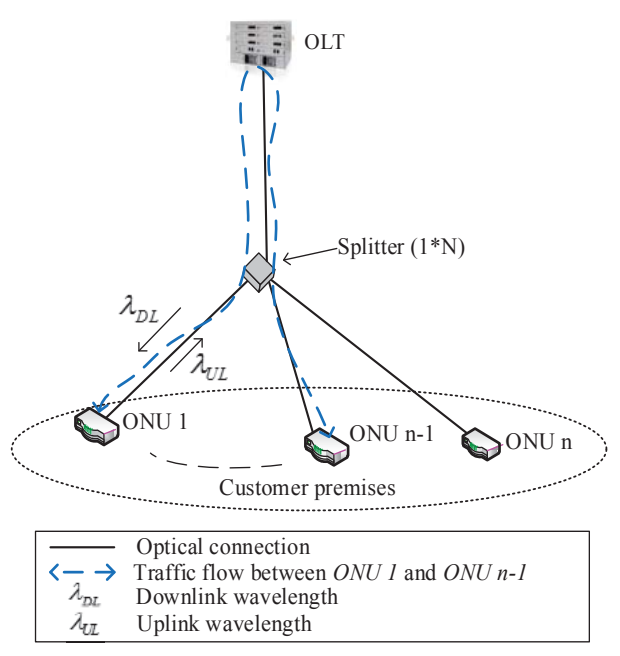

Fig. 2. System model for TDM-PON based EL-TTBI scheme with sleep mechanism.

TABLE I

EXAMPLE OF ONUS' GROUP FORMATION

\begin{tabular}{|c|l|c|}
\hline Destination ONU & \multicolumn{1}{|c|}{ Source ONU } & $D_{r e q}$ \\
\hline ONU 1 & ONU 2, ONU 3, ONU 5, ONU 7 & $D_{1}$ \\
\hline ONU 2 & ONU 1, ONU 3, ONU 6 & $D_{2}$ \\
\hline ONU 3 & ONU 1, ONU 2 & $D_{1}$ \\
\hline ONU 4 & ONU 7 & $D_{3}$ \\
\hline ONU 5 & ONU 2, ONU 3 & $D_{1}$ \\
\hline ONU 6 & ONU 1, ONU 3 & $D_{3}$ \\
\hline ONU 7 & ONU 4 & $D_{2}$ \\
\hline
\end{tabular}

\section{B. Proposed Working Procedures for EL-TTBI Scheme:}

In this sub-section, we explain how our proposed EL-TTBI scheme works. As mentioned earlier that our prime objective in this paper is to minimize ONUs' energy consumption using sleep mode mechanism while meeting the delay requirements of local customer internetworking traffic (facilitate inter-ONU communication through the OLT). Note that an ONU could send traffic to several ONUs. If there are $N$ number of ONUs in a TDM-PON, the maximum number of destination ONUs could be $N-1$ for a source ONU. Similarly, a particular ONU could be a destination node for $N-1$ number of ONUs. In our solution, the OLT may need to manage one or more groups considering ONUs' traffic sharing and desired delay requirements at a given time. Here, we explain how the OLT forms groups of ONUs in our proposal.

1) ONUs' group formation: During each listening interval of an ONU, an ONU can notify the OLT its destination ONUs (to whom the ONU has traffic to forward). We now use the following example to explain how the OLT forms group based on the received requests from different ONUs during their listening intervals. Suppose that in a TDM-PON there are 7 ONUs (for simplicity we consider here smaller number of ONUs) and the OLT supports 3 levels of delay requirements $\left(D_{r e q}\right)$. Assume that the OLT prepares a summarized information presented in Table I considering ONUs' requests. Then,

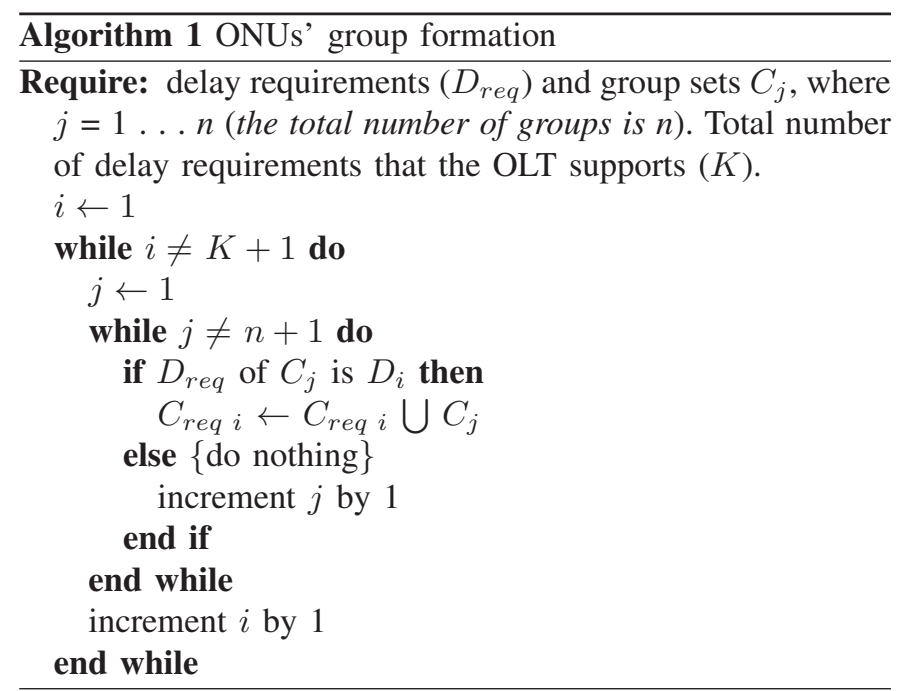

the OLT generates 7 sets: $C_{1}=\{$ ONU 1, ONU 2, ONU 3, ONU 5, ONU 7 $\}, C_{2}=\{$ ONU 1, ONU 2, ONU 3, ONU 6 $\}$, $C_{3}=\{$ ONU 1, ONU 2, ONU 3$\}, C_{4}=\{$ ONU 4, ONU 7 $\}, C_{5}$ $=\{\mathrm{ONU} 2, \mathrm{ONU} 3, \mathrm{ONU} 5\}, C_{6}=\{\mathrm{ONU} 1, \mathrm{ONU} 3, \mathrm{ONU} 6\}$, and $C_{7}=\{\mathrm{ONU} 4, \mathrm{ONU} 7\}$. Then, the sets having the same delay requirement are merged together. In this example (see Table I), $C_{1}, C_{3}$ and $C_{5}$ have the same delay requirement $D_{1}$. Similarly, $C_{2}$ and $C_{7}$ demand delay requirement $D_{2}$. Finally, $C_{4}$ and $C_{6}$ need delay requirement $D_{3}$. Then the new sets that require delay requirement $D_{1}, D_{2}$ and $D_{3}$ are $C_{r e q 1}, C_{r e q 2}$ and $C_{r e q 3}$, respectively. And the elements of $C_{r e q 1}, C_{r e q 2}$ and $C_{r e q 3}$ are $\{$ ONU 1, ONU 2, ONU 3, ONU 5, ONU 7\}, \{ONU 1, ONU 2, ONU 3, ONU 4, ONU 6, ONU 7\}, and \{ONU 1, ONU 3, ONU 4, ONU 6, ONU 7\}, respectively. The aforementioned procedures are presented in Algorithm 1.

2) ONUs' group sleep management: Here, we present how all ONUs belong to the same set (e.g. ONUs in set $C_{r e q 1}$ require $D_{1}$ ) manage sleep mechanism. At this point, we assume that delay requirement $D_{i}<D_{i+1}$, where $i=1, \ldots K$ (the total number of delay requirements OLT supports = $K)$. Additionally, in our scheme, we consider that all delay requirement values are multiples of the most stringent delay requirement. In our proposal, the sleep interval length for ONUs that need a particular level of delay requirement (e.g. $\left.D_{1}\right)$ is calculated as follows:

$$
T_{\text {sleep }}=D_{i}-2 \times T_{t r}-T_{p r},
$$

where $T_{t r}$ is the transmission delay between an OLT and ONU, and $T_{p r}$ is the time required for the OLT to process source ONU's traffic and forward them to the destination ONU.

We now use the above example to describe how the OLT manages sleep mechanism for a particular group of ONUs having the same delay requirement. Considering delay requirement $D_{1}$, the OLT calculates sleep interval length $T_{\text {sleep }}$ using (1) for the ONUs of set $C_{r e q 1}$ and notifies $T_{\text {sleep }}$ to all the ONUs of $C_{r e q 1}$. Then, ONUs of $C_{r e q 1}$ which do not have traffic to transmit and/or receive move into sleep mode. Note that, in our solution, $T_{\text {sleep }}$ is the maximum 


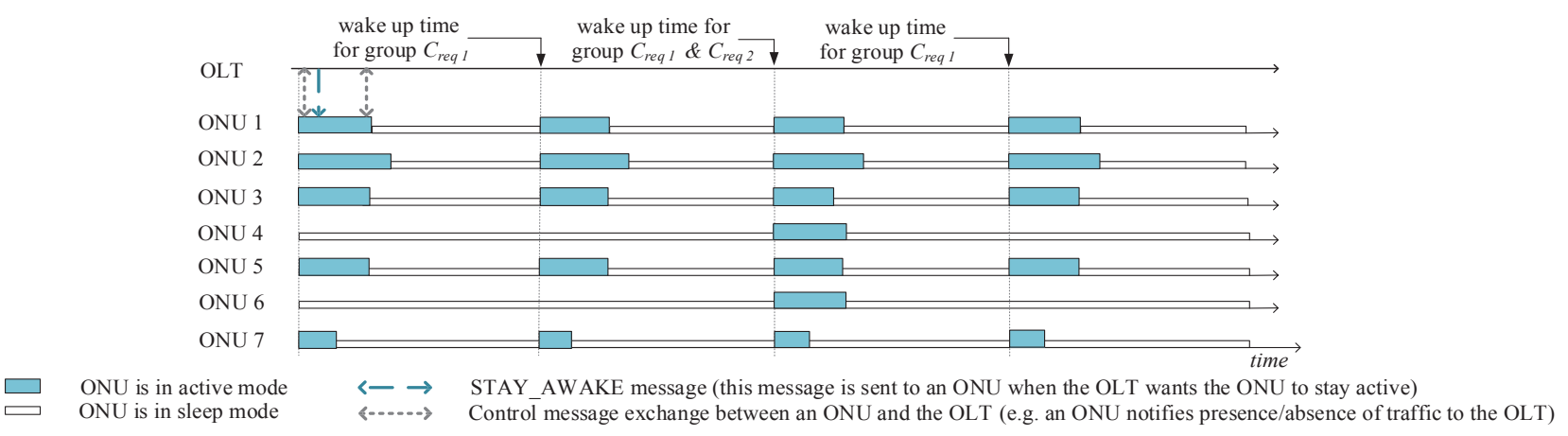

Fig. 3. Sleep management of ONUs belong to different groups having different delay requirements.

amount of time an ONU of a group is allowed to sleep. After $T_{\text {sleep }}$, all the ONUs of that set return to active mode and notify the OLT if they have traffic to forward (an ONU's notification includes the address of destination $\mathrm{ONU}(\mathrm{s})$ and the amount of uplink bandwidth it requires to forward those traffic). After sending notifications, ONUs wait for the OLT's instruction. The OLT invokes ONU(s) to move into sleep mode when there is no traffic to share. Conversely, the OLT sends notification messages (i.e. STAY_AWAKE message, see Fig. 3) to the ONUs which has traffic to receive and/or transmit mentioning allocated bandwidth for them. Note that the OLT cannot forward unicast traffic to all of the ONUs of a group at the same time. Therefore, the OLT serves all the ONUs of a group that have traffic to receive one after another. Similarly, the OLT allocates dedicated uplink slot to each of the ONUs which have traffic to transmit. In the similar manner, the OLT manages all other groups (e.g. $C_{r e q 2}, C_{r e q 3}$ ). Figure 3 depicts how the OLT manages sleep mechanism for different ONU groups (for the sake of simplicity, this figure describes sleep management for $C_{r e q 1}$ and $C_{r e q 2}$ only of our example).

At this point, it is important to mention here that in SIEPON standard [21] ONUs' synchronized wake-up concept is proposed. According to this standard, ONUs joining for same multicast or broadcast traffic should wake-up together. We also have synchronous wake-up concept in our proposal. However, our solution is different in several aspects. First, the major difference between [21] and our proposal is that our synchronous wake-up approach is used for sharing local customer internetworking traffic (this traffic could be unicast, multicast or broadcast). Secondly, our proposal defines how sleep interval length of ONUs belong to a group can be defined for a particular delay requirement. Furthermore, we define how an OLT could manage several ONU groups considering different traffic delay requirements. Finally, in our proposal, the ONUs of a group which have traffic to share remains active after switching from sleep mode. Whereas, all other ONUs of that group that do not have any traffic move into sleep mode.

\section{PERFormance AnAlysis}

We use our earlier developed TDM-PON module [19], designed in OPNET modular to analyze TDM-PONs' energy efficiency and traffic performance. We compare our proposed solution with cyclic sleep, where an ONU periodically enters into sleep mode or remains in active mode based on presence or absence of its uplink and downlink traffic [20]. Here, we assume that all traffic source and destination is within the PON. It is important to mention here that our proposed ELTTBI scheme is still under development. Therefore, in this paper, we present our initial findings. Here, we are interested in measuring end-to-end delay experienced by packets among customers connected with different ONUs and ONUs' energy saving performance. In particular, we assume that at a given time the number of sender customers is 6 and recipient customer is 1 . In addition, we consider that ONUs that serve these customers belong to the same group. For performance evaluation, we consider that the sender customers forward VBR traffic to the recipient customer (we consider ON/OFF source model for VBR traffic generation in our simulation, similar to [3]).

To evaluate energy saving performance of EL-TTBI and cyclic sleep scheme, we consider that an ONU's power consumption in sleep and active mode is $4.69 \mathrm{~W}$ and 1.28 $\mathrm{W}$, respectively [17]. When, an ONU moves into sleep to active mode, it requires $125 \mu$ s transition time [17]. Additionally, we consider that the transmission delay between the OLT and an ONU is $0.2 \mathrm{~ms}$ [3]. We evaluate performance of the proposed EL-TTBI and cyclic sleep scheme under three different delay requirements $\left(D_{\text {req }}\right)$ (i.e. $4 \mathrm{~ms}, 10 \mathrm{~ms}$ and $20 \mathrm{~ms}$ ). For simplicity of representing the performance improvement due to using synchronous ONUs group wakeup for local customer internetworking in our proposal, we assume that in both schemes the OLTs uses (1) to calculate sleep interval lengths for ONUs. In addition, we quantify energy consumption results of ONUs under each scheme as the portion of energy consumption of the solution where an ONU always stays active (e.g. IEEE 802.3ah [9]).

Figure 4 (a), (b) and (c) compare the end-to-end traffic delay Cumulative Distributed Function (CDF) results for $D_{\text {req }}$ $4 \mathrm{~ms}, 10 \mathrm{~ms}$ and $20 \mathrm{~ms}$, respectively. Results show that in all three scenarios our proposed solution preforms better than cyclic sleep based solution (in proposed EL-TTBI, for all 


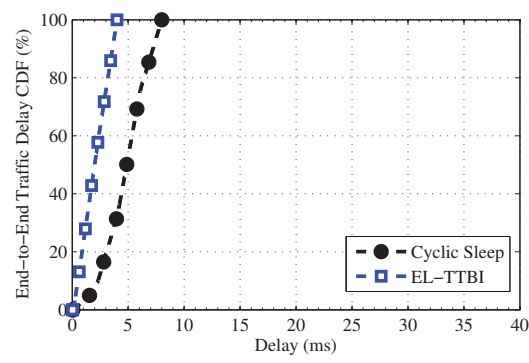

(a)

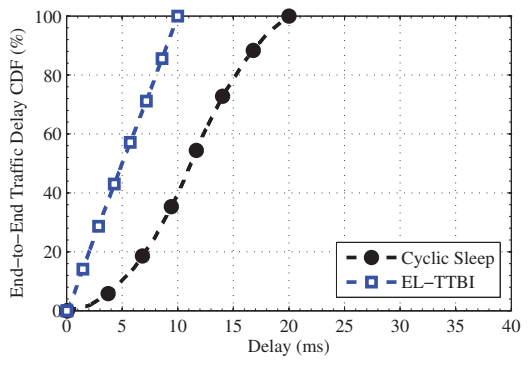

(b)

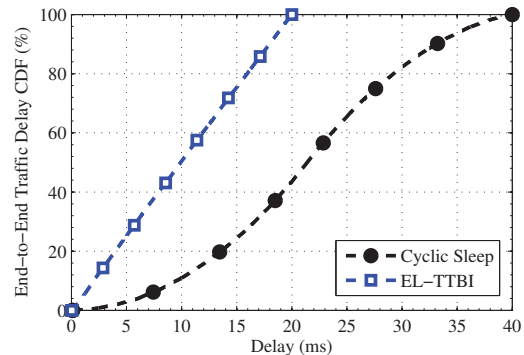

(c)

Fig. 4. Comparison of end-to-end traffic delay CDF results of EL-TTBI and cyclic sleep scheme: (a) $D_{r e q}=4 \mathrm{~ms}$; (b) $D_{r e q}=10 \mathrm{~ms}$; (c) $D_{r e q}=20 \mathrm{~ms}$.

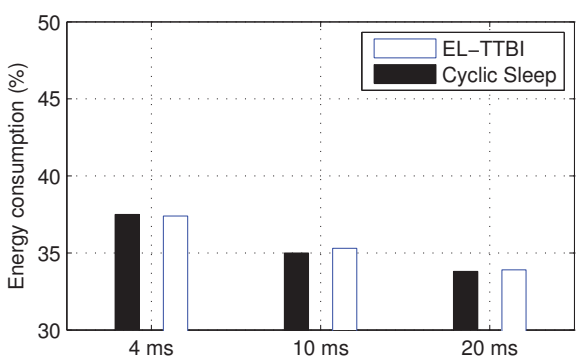

Fig. 5. Energy consumption performance comparison between two proposals.

delay requirements $100 \%$ of traffic meet delay requirement). This happens because in our solution, the ONUs that have traffic to share manage a group and wake up from sleep mode together to share their traffic if there is any. Whereas, in case of cyclic sleep based solution, delay performance is worse than proposed EL-TTBI as ONUs in this solution do not have any coordination when they have traffic to share. Finally, from Fig. 5 we can appreciate that proposed EL-TTBI presents almost similar energy saving performance in all cases compared to cyclic based solution.

\section{CONCLUSiOn}

In this paper, we presented an energy efficient and latency aware local customer internetworking scheme. Here, we have presented the initial findings of our study. Simulations results depict that the proposed solution not only meets $100 \%$ delay requirement of traffic but also shows satisfactory energy saving performance compared to the cyclic sleep scheme.

\section{ACKNOWLEDGMENT}

This work was supported by grant No. EEWS-2014N01140044 from Climate Change Research Hub Project of the KAIST EEWS Research Center (EEWS: Energy, Environment, Water and Sustainability).

\section{REFERENCES}

[1] Data and Internet of Things: A Roadmap for Smart Environments, 1st ed., Springer Cham Heidelberg New York Dordrecht London, 2014, pp. 169-187.

[2] M. Gruber et al., "Earth-2014 energy aware radio and network technologies," in Personal, Indoor and Mobile Radio Communications, 2009 IEEE 20th International Symposium on, Sept 2009, pp. 1-5.
[3] S. S. Newaz et al., "Adaptive delay-aware energy efficient TDM-PON," Computer Networks, vol. 57,no. 7, pp. 1577-1596, 2013.

[4] F. Bonomi et al., "Fog Computing and Its Role in the Internet of Things," in Proceedings of ACM MCC, 2012, pp. 1316.

[5] J. G. Kim et al., "Local customer internetworking scheme in passive optical networks," Advanced Communication Technology, 2009. ICACT 2009, vol. 03, no. 5, pp. 1759-1761, 2009.

[6] Nadarajah et al., "Protection Switching and Local Area Network Emulation in Passive Optical Networks," IEEE Journal of Lightwave Technology, vol. 24, no. 5, pp. 1955-1967, 2006.

[7] J. G. Kim et al., "Mini-Slot-Based Transmission Scheme for Local Customer Internetworking in PONs," ETRI Journal, vol. 30, no. 2, pp. 282-289, 2008.

[8] P. YunFeng, "Optical local area network emulations over Ethernet passive optical networks: A survey," in Science in China Series F: Information Sciences, vol. 51, pp.1000-1009, 2008.

[9] IEEE Std. 802.3ah-2004, "Part 3: Carrier Sense Multiple Access with Collision Detection (CSMA/CD) Access Method and Physical Layer Specifications," Institute Computer Society, 7 Sept. 2004.

[10] A.V. Tran, et al., "Bandwidth-efficient PON system for broad-band access and local customer internetworking," Photonics Technology Letters, IEEE, vol. 18, no. 5, pp. 670-672, March 2006.

[11] IEEE Std 802.3av-2009, "Physical layers Specifications and Management Parameters for $10 \mathrm{~Gb} / \mathrm{s}$ Passive Optical Networks," October 2009.

[12] Huawei Unveils World's First 40G PON Prototype. Oct. 2011. [Online] Available: http://pr.huawei.com/en/news/hw103292.htm.VLOV3yvF87k.

[13] J.K. Zao et al., "Pervasive brain monitoring and data sharing based on multi-tier distributed computing and linked data technology," Frontiers in Human Neuriscience, vol. 8, Article 370, June 2014.

[14] Technology White Paper, Alcatel/Lucent, Smart Choices for the Smart Grid. Retrieved from the web. Feb. 2011. [Online]

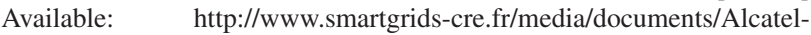
Lucent_SmartChoicesfortheSmartGrid_TWP_EN.pdf.

[15] S. S. Newaz et al., "A web based energy cloud platform for campus smart grid for understanding energy consumption profile and predicting future energy demand," International Conference on Information and Communication Technology Convergence (ICTC), pp.173,178, 22-24 Oct. 2014.

[16] P. Kansal et al., "Bandwidth and Latency Requirements for Smart Transmission Grid Applications," Smart Grid, IEEE Transactions on , vol.3, no.3, pp.1344,1352, Sept. 2012.

[17] Shing-Wa Wong et al., "Sleep Mode for Energy Saving PONs: Advantages and Drawbacks," GLOBECOM Workshops, 2009 IEEE , pp.1,6, Nov. 30 2009-Dec. 42009.

[18] R. Kubo, et al., "Study and demonstration of sleep and adaptive link rate control mechanisms for energy efficient 10GEPON," IEEE/OSA J. Opt. Commun. Net., vol. 2, Issue 9, pp. 716-729, 2010.

[19] M. A. F. Yousif et al., "Modeling and simulation of EPON with sleep mode enabled using OPNET," International Conference on Information and Communication Technology Convergence (ICTC), pp.16,21, 22-24 Oct. 2014.

[20] ITU-T Recommendation G Suppl. 45, Supplement on GPON, Power Conservation, May 2009.

[21] IEEE Std 1904.1-2013, "IEEE Standard for Service Interoperability in Ethernet Passive Optical Networks (SIEPON),’ pp.1,834, Sept. 202013. 